\title{
Two-arm Robot Teleoperation using a Multi-touch Tangible User Interface
}

\author{
Andreas Angerer, Andreas Bareth, Alwin Hoffmann, Andreas Schierl, Michael Vistein \\ and Wolfgang Reif \\ Institute for Software and Systems Engineering, University of Augsburg, D-86135 Augsburg, Germany
}

\begin{abstract}
Keywords: Software Architecture, Teleoperation.
Abstract: Teleoperation of robots can be interesting in various scenarios: operation in hazardous environments, medical surgery in the presence of radiation or, in general, remote control of robots in (partially) unknown environments. Crucial to teleoperation systems is an intuitive interface for the remote operator to ensure straightforward and precise control of the operated system. Advanced hardware devices and powerful software frameworks allow for quick development of such interfaces. We present a case study implementing a novel two-arm robot teleoperation interface using multi-touch and tangible user interface concepts. The focus lies on the implementation of this system, using an object oriented robot programming framework and the Microsoft Surface as a user interface platform.
\end{abstract}

\section{INTRODUCTION}

Recent progress in sensor hardware, sensor information processing and cognititive capabilities led to a significant increase in the autonomy of (mobile) robot systems. Robots can autonomously fetch objects (Jain and Kemp, 2010), open doors (Meeussen et al., 2010), and even cook pancakes (Beetz et al., 2011). While this progress leads to less involvement of human operators in some areas, there are other cases where human intervention is desirable or even indispensable e.g. in rescue robotics, where certain decisions should rather be taken by a human than relying on a machine. In these areas, teleoperation systems that support human operators in efficiently controlling robot systems remotely play an important role.

A variety of teleoperation systems has already been developed, mostly targeted at controlling the navigation of mobile robots. In (Skrzypczyliski, 1997), a graphical interface has been developed to achieve telepresence for an operator navigating a mobile robot through indoor environments. A similar concept is employed in (Zalud, 2006) with a special focus on data fusion of various sensors to augment the picture taken by the robot's main camera. In both projects, visual feedback by cameras as well as $3 \mathrm{D}$ visualization of the scenery play an important role.

Besides different concepts for presenting data to the operator, new user interface concepts like multitouch have also been applied to robot teleoperation.
Micire et al. (Micire et al., 2011) presents a system based on the Microsoft Surface for remote operation of a mobile robot through an intuitive multi-touch interface. In (Seifried et al., 2009), a multi-touch tabletop device is used for controlling several home devices, including a vacuum cleaner robot. In those systems, the intuitiveness of (multi-)touch user interfaces is used for easing the remote operation.

In this work, we present a multi-modal, tangible user interface for teleoperation of a two-arm robot system. We developed this system mainly as a case study to evaluate the usefulness of an object oriented robot application framework called the Robotics API (cf. (Angerer et al., 2010)); the experiences made will be illustrated in this work. Besides that, this system could serve as an intuitive way to operate a mobile two-arm robot which is currently planned to be built at our institute. On the hardware side, two KUKA Light Weight Robot (LWR) arms were used. These 7-axis robot arms achieve industry-grade movement precision, and integrated torque sensors and corresponding control algorithms (Bischoff et al., 2010) open up interesting possibilities e.g. for forcecontrolled manipulation. Those sensors can, however, also provide valuable feedback in a teleoperation scenario, as will be illustrated later. For realizing the teleoperation user interface, we employed the Microsoft Surface platform ${ }^{1}$ and the corresponding SDK.

\footnotetext{
${ }^{1}$ http://www.microsoft.com/surface/
} 


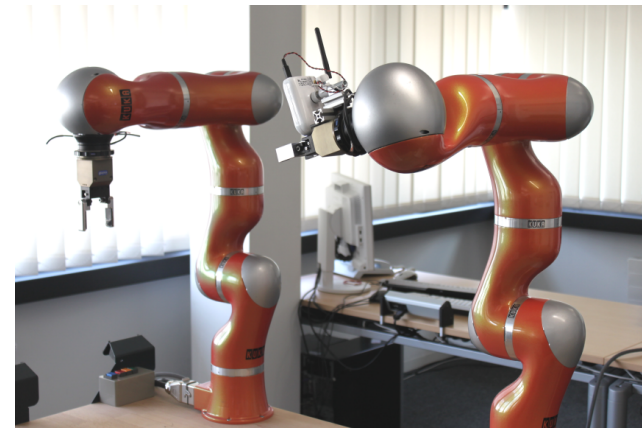

Figure 1: Light Weight Robot setup.

the camera (and thus the scene perceived by it) corresponds directly to the arm movement commanded by the user. It can be considered a "first person" perspective. In the observer mode, the robot arm with the camera mounted takes an observer position, while the second robot arm, the manipulator robot, performs the actual task (cf. Fig. 2). The observer robot automatically follows movements of the manipulating robot. The operator can rotate the camera robot around the observed point of interest. Additionally, by moving the observer robot closer to or further away from the point of interest, a zooming functionality can be realized. Thus, the scene can be observed flexibly.

The paper is structured as follows: In Sect. 2, the concepts and user interface of the developed teleoperation application are explained. Sect. 3 explains the implementation challenges and how those could be adressed using the Robotics API. The following Sect. 4 presents some experimental results. Finally, Sect. 5 concludes the work and gives an outlook.

\section{A TANGIBLE USER INTERFACE FOR TELEOPERATION}

This work is focused on a rather simple test scenario: A tele-operator should be able to pick workpieces from some location and put those workpieces to any target location. Though the core steps in this task are quite simple, it may involve preliminary steps like deciding a gripping strategy (see (Siciliano and Khatib, 2008), pp. 671) or even moving other objects that block access to the workpiece to be transported. A human operator can infer most of those necessary steps quickly from just observing the scene (or the camera picture, respectively), in contrast to complex cognition steps in an automated robotic system. The tele-operator should be able to perform pick and place tasks with intuitive support by the teleoperation system. Similar to previous work in this area (see Sect. 1), visual perception of the scene is considered very important and should be realized here by a single camera, mounted next to the gripper of one of the robot arms. Fig. 1 shows the laboratory setup of both Light Weight Robots. The observer robot is equipped with a simple network camera (Intellinet NSC15-WG), which utilizes Wireless LAN for transmitting the video stream to the application.

The teleoperation system supports two distinct modes of operation: In the direct control mode, the robot arm with the camera mounted on it performs the gripping task. In this mode, the movement of

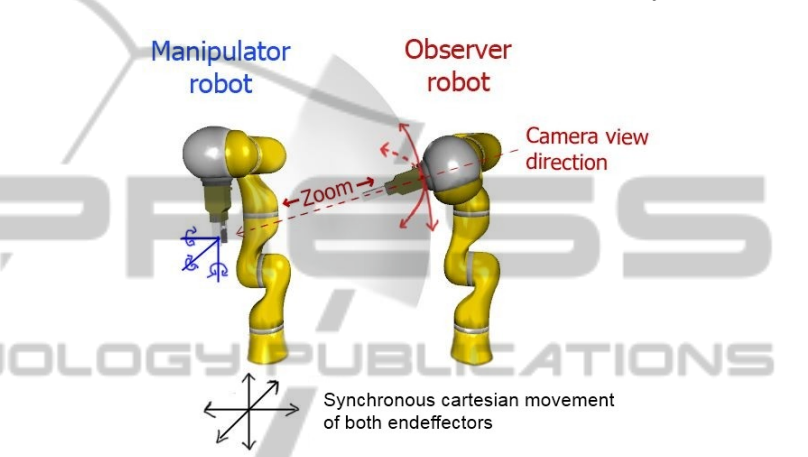

Figure 2: Observer mode.

As mentioned before, the user interface of the proposed teleoperation system is based on the Microsoft Surface platform. This platform consists of a tableshaped, touch-sensitive 30-inch display and a corresponding Software Development Kit. In contrast to many touch-sensitive devices nowadays, the Microsoft Surface utilizes optical recognition of fingers or other objects that touch it. Therefore, arbitrary shapes can be recognized, as well as special graphical tags.

The application designed for teleoperation on the Microsoft Surface offers a user interface consisting of four main parts as shown in Fig. 3. The camera picture (1) is shown in the top center part of the user interface area, where it is in the center of the operator's field of view. Below the camera view, a $3 D$ view (2) of the robots is located. The view can be rotated and zoomed in order to get a good overview of the robots' poses. It listens to data supplied by the robots' joint position sensors and updates the pose visualization accordingly. The perspective can be rotated by swiping over the view with one finger, and zoomed by swiping two fingers in opposite directions. Additionally, pre-defined perspectives on the scene can be activated by pressing one of the buttons for Left, Top, Right, Front or Back perspective (cf. Fig. 4). The view also displays the Cartesian forces measured at the robots' endeffectors, which are visualized as ar- 


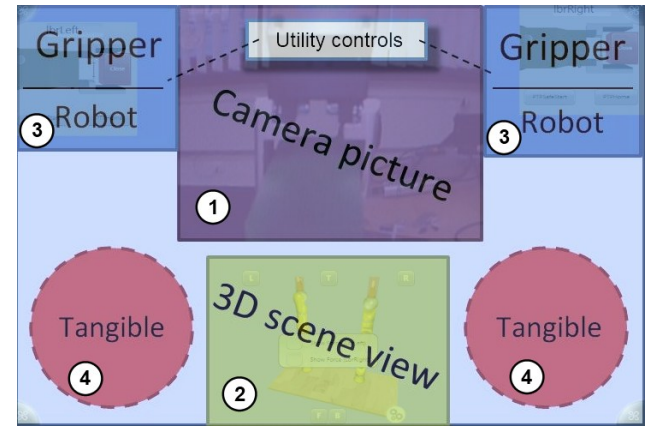

Figure 3: Overview of the tangible teleoperation user interface.

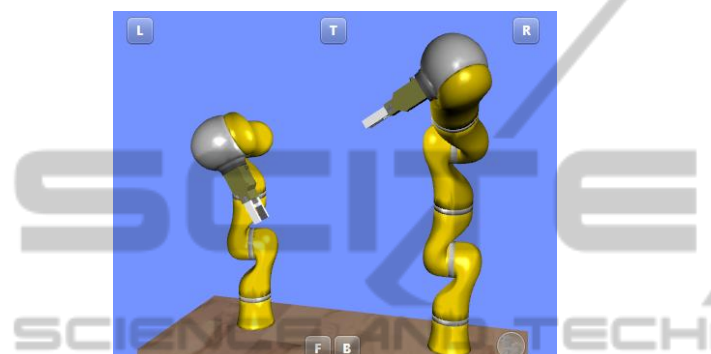

Figure 4: 3D visualization of robot poses.

rows pointing in the direction of the measured force. The length of the arrows indicates the magnitude of the force. This proved to be valuable feedback for the operator to judge the current state of the system.

For controlling the robot arms during teleoperation, a combination of physical objects and virtual user interface elements is used. The motion control of the manipulator robot is realized using the $3 \mathrm{D}$ mouse SpaceNavigator by 3Dconnexion. This 6-DOF mouse can be used to intuitively control cartesian movement and rotation of the robot endeffector. The SpaceNavigator is at the same time used as a tangible user interface element: When it is placed somewhere on the teleoperation UI (4), a Surface tag sticked to its bottom is recognized by the application. The user interface control shown in Fig. 5 is displayed and at the same time, movement of the manipulator robot arm is enabled. From then on, the SpaceNavigator controls the robot movement. The displayed control provides additional selectable options, in particular switching from direct mode to observer mode and vice versa.

In observer mode, the observing robot arm can be rotated around the manipulator robot's endeffector in two dimensions (up/down and left/right). Additionally, the user can zoom into and out of the perspective by moving the observer robot closer to or further away from the manipulator robot. This three degrees of freedom are controlled by a second tangible interface element, which is a simple hemispherical object with a Surface tag attached to it. Upon contact to the

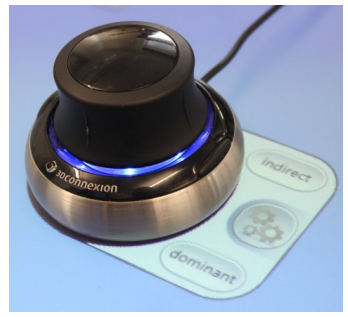

Figure 5: Visualization of the manipulator robot controller.

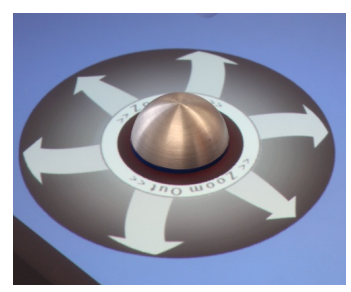

Figure 6: Visualization of the observer robot controller.
Surface, the UI control shown in Fig. 6 is displayed. Moving the tangible element outside the center of the visualization rotates the observer robot, while rotating the tangible element when it is inside the center zone controls the zoom level by moving the observer robot closer to or away from the manipulator robot's tool

The UI elements depicted as (3) in Fig. 3 provide some utility controls for the robots (e.g. moving to safe positions) and grippers.

\section{IMPLEMENTATION CHALLENGES AND CONCEPTS}

The teleoperation application as described in the previous section posts the following requirements to the underlying robot control framework:

1. Reading Sensor Data: information about the current robot positions and the measurements of the torque sensors have to be provided.

2. Cartesian Velocity Control: for the proposed 6DOF mouse and tangible input devices, control of the Cartesian velocity of the robot arms is most adequate. Thus, means of specifying Cartesian velocity set points are required.

3. Movement Synchronization: in observer mode, the movement of the observer robot needs to be synchronized to the manipulator robot's movement. Note that the observer robot may itself be moving with a certain velocity relative to the manipulator robot (i.e. when changing the observer perspective), so both requirements need to be combinable.

The application was implemented using the Robotics API (cf. (Angerer et al., 2010)), an objectoriented robot control framework developed in the research project SoftRobot. It is a modular framework for developing real-time critical robotic applications. It supports the development of complex multirobot applications, including real-time robot coopera- 

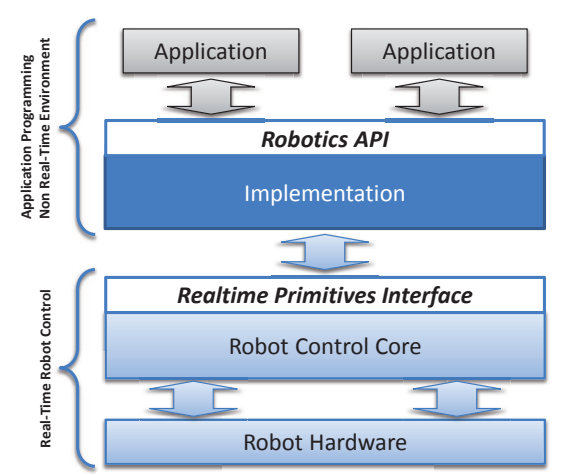

Figure 7: Robot control architecture used for implementation of the teleoperation system.

tion and sensor-controlled manipulation. The framework's architecture consists of two main parts (cf. Fig. 7), the Robotics Application Programming Interface (Robotics API), and a Robotics Control Core (RCC) which provides the Realtime Primitives Interface (RPI, see (Vistein et al., 2010)). The core idea behind this architecture is a splitting of concerns when developing robotic applications: the application workflow can be developed using the object-oriented Robotics API which provides a set of concepts to support the developer, like classes encapsulating manipulator or tool functionality, classes for modeling frames and physical objects and classes for accessing and processing sensor data. Actuator operations, reactions to defined events and actuator synchronization can be defined by means of Robotics API concepts. Subsequently, they are automatically mapped to an RPI dataflow command specification and transferred to the Robotics Control Core, which ensures real-time critical execution of those requests. In that way, real-time robot control is encapsulated and separated from high-level application workflow.

The Robotics API provides means of reading a variety of sensor data which matches the first requirement of the teleoperation application. Reading sensor data is performed by registering SensorListener objects to instances of the Sensor class. The measurement values delivered by a respective driver module in the RCC are then automatically propagated to the registered listeners.

Controllable Devices like robot arms are modeled by specialized versions of the Robotics API class $A c$ tuator. Actuators have the ability to execute certain Actions, like joint or Cartesian motions (for robots) or gripper commands (for grippers). Instances of the class Robot, which is a subclass of Actuator, also support the execution of Actions that accept velocity set points as input during their execution. One such Action is called CartesianJogging. Its implementation employs an online planner and ensures that the spec-

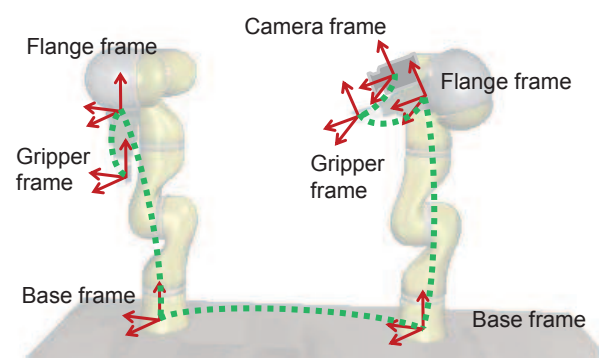

Figure 8: Relevant frames for CartesianJogging in teleoperation. Dotted lines indicate connections between frames.

ified cartesian translational and rotational velocities are established by the robot's endeffector in a continuous way. The CartesianJogging action is used in the teleoperation application to achieve the desired cartesian velocity control by mapping deflections of the 6-DOF mouse and movement of the observer robot tangible control to target velocity values.

Considering movement synchronisation, the Robotics API provides some built-in support by its world model. Cartesian coordinate systems, modeled by the Frame class, may be moving relative to other frames (like a robot's endeffector frame relative to its flange frame) and Action implementations are provided information about the current state (position, velocity) of moving frames and have to respect this. In particular, CartesianJogging requires two Frames as parameters: The reference frame defines the base frame relative to which the jogged frame should be moving with the specified velocity. If the reference frame is itself moving, the Action's implementation ensures that the motion is followed in addition to the specified jogging velocity. This feature is useful for the application's observer mode: here again, a CartesianJogging Action can be used with an appropriate choice of the reference frame. Fig. 8 shows all frames that are relevant in the teleoperation application.

One problem remains considering the zooming functionality of the observer robot: During zooming, the center of rotation of the observer robot's CartesianJogging should stay in the position of the manipulator robot's endeffector. This was not the case with the existing implementation of CartesianJogging, which used the jogged frame's position as rotation center (i.e. the observer robot's camera frame in this case). To solve this problem in a generic way, we extended the definition of the CartesianJogging Action by introducing a third frame, called the pivot frame. This frame's position is used as point at which to apply rotational jogging velocities. By choosing the manipulator robot's endeffector frame as pivot frame for the observer robot's jogging Action, the behavior is as desired. 


\section{EXPERIMENTAL RESULTS}

The developed teleoperation system was successfully tested using two Lightweight Robot arms mounted on a table. For a first validation of its effectiveness, we put up a test scenario consisting of several objects randomly placed in a box. The task was to pick a certain object from the box. Such challenges arise in practical teleoperation scenarios as well, like handling contaminated material in nuclear power plants, or searching for survivors in destroyed buildings. In most cases, multiple actions (moving away blocking objects) were necessary to get access to the desired object. The operators (students, postgraduates and professors with technical background) had no eye contact to the robots, they had to stick to the information provided by the teleoperation system. After a short learning phase, they could all complete the task successfully. The observer mode was the preferred mode of operation, as it proved to be more flexible due to the ability to inspect the scene from various perspectives. Although the task of moving objects seems quite simple, the experiments showed that quite some visual and haptic information is required to correctly judge the geometric properties and relations of the involved objects.

Fig. 9 shows a scene from the experiments. Fig. 10 shows the same scene viewed from a different angle by adjusting the observer robot. This example shows how the ability to change perspective helps in judging the situation: From just observing the first, central perspective on the scene, the operator cannot recognize whether the robot's gripper is positioned above the hole-puncher that should be fetched from the box. By rotating the perspective, the situation becomes clearer. The visualization of the contact force as shown in Fig. 11 offers the operator an additional perceptional dimension and can help to resolve visual ambiguities. A video of the application is available ${ }^{2}$.

\section{CONCLUSIONS}

In this work, we presented an application for teleoperation of a two-arm robot system. The realization employs multi-touch and tangible user interface concepts and is realized on the Microsoft Surface platform. The implementation of the teleoperation system concentrated mainly on the user interface design and the general application logic. Interfacing the robot arms showed to be straightforward and simple due to the rich framework support provided by the Robotics

\footnotetext{
${ }^{2} \mathrm{http} / / /$ video.isse.de/teleop
}

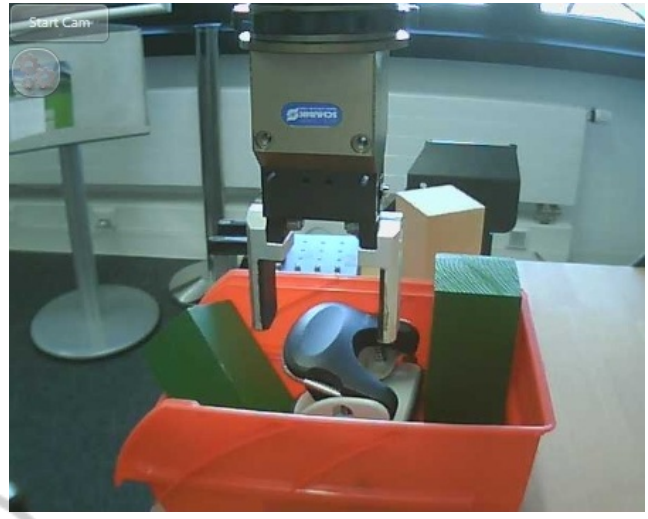

Figure 9: Manipulated object viewed from a central perspective.

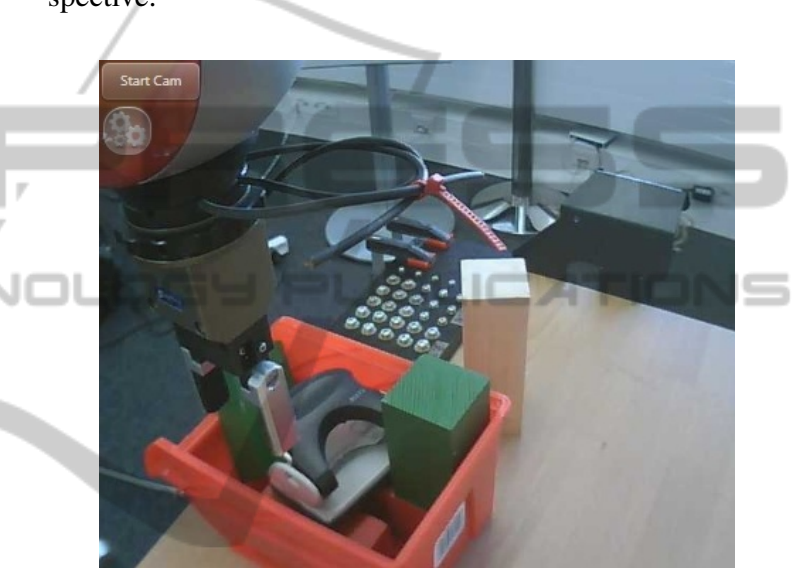

Figure 10: Manipulated object viewed from a side perspective.

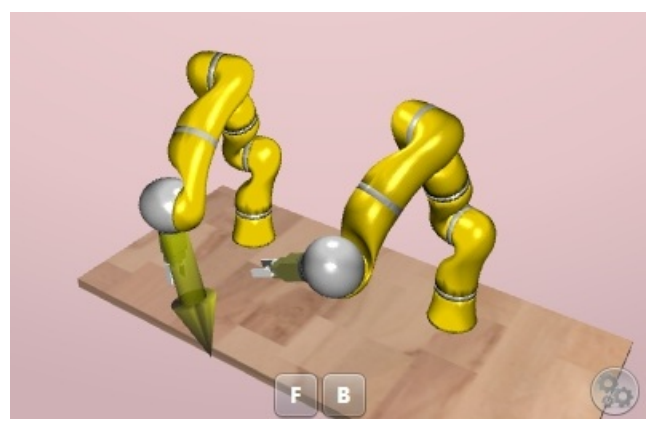

Figure 11: Force visualization during contact to the object.

API for controlling the arms as well as monitoring the various sensors. The CartesianJogging action proved to be a powerful tool for creating a flexible, velocitybased robot control interface. However, we learned that its initial definition using two frames did not cover all practical cases of expected behavior, so we had to extend it by introducing an additional frame.

Currently, we are working on some extensions to the current implementation to make the system more robust. In the current state, reaching a robot's joint 
limits leads to termination of the jogging action and requires it to be restarted. Similar problems arise in singular robot poses. First tests with strategies that limit joint velocities in such situations look very promising.

In the future, we will continue to create case studies like this one, as we see them as an excellent option for evaluating the current state of a software framework. Beyond that, the developed teleoperation system could be a valuable tool for controlling e.g. a planned mobile two-arm manipulator.

\section{ACKNOWLEDGEMENTS}

This work presents results of the research project SoftRobot which was funded by the European Union and the Bavarian government within the HighTech-Offensive Bayern. The project was carried out together with KUKA Laboratories $\mathrm{GmbH}$ and MRK-Systeme GmbH and was kindly supported by VDI/VDE-IT. NI $=4 \mathrm{NI}$
Intelligent User Interfaces, IUI '11, pages 145-154, New York, NY, USA. ACM.

Seifried, T., Haller, M., Scott, S. D., Perteneder, F., Rendl, C., Sakamoto, D., and Inami, M. (2009). CRISTAL: a collaborative home media and device controller based on a multi-touch display. In Proc. ACM Int. Conf. on Interactive Tabletops and Surfaces, ITS '09, pages 33-40, New York, NY, USA. ACM.

Siciliano, B. and Khatib, O., editors (2008). Springer Handbook of Robotics. Springer, Berlin, Heidelberg.

Skrzypczyliski, P. (1997). Supervision and teleoperation system for an autonomous mobile robot. In Proc. 1997 IEEE/RSJ Int. Conf. on Intelligent Robots and Systems, volume 2, pages 1177 -1181 vol.2.

Vistein, M., Angerer, A., Hoffmann, A., Schierl, A., and Reif, W. (2010). Interfacing industrial robots using realtime primitives. In Proc. 2010 IEEE Intl. Conf. on Automation and Logistics (ICAL 2010), Hong Kong, China, pages 468-473. IEEE.

Zalud, L. (2006). ARGOS - system for heterogeneous mobile robot teleoperation. In Proc. 2006 IEEE/RSJ Int. Conf. on Intelligent Robots and Systems, pages 211 -216 .

\section{REFERENCES}

Angerer, A., Hoffmann, A., Schierl, A., Vistein, M., and Reif, W. (2010). The Robotics API: An objectoriented framework for modeling industrial robotics applications. In Proc. 2010 IEEE/RSJ Intl. Conf. on Intelligent Robots and Systems (IROS 2010), Taipeh, Taiwan, pages 4036-4041. IEEE.

Beetz, M., Klank, U., Kresse, I., Maldonado, A., Mösenlechner, L., Pangercic, D., Rühr, T., and Tenorth, M. (2011). Robotic roommates making pancakes. In 11th IEEE-RAS International Conference on Humanoid Robots, Bled, Slovenia.

Bischoff, R., Kurth, J., Schreiber, G., Koeppe, R., AlbuSchäffer, A., Beyer, A., Eiberger, O., Haddadin, S., Stemmer, A., Grunwald, G., and Hirzinger, G. (2010). The KUKA-DLR lightweight robot arm - a new reference platform for robotics research and manufacturing. In Proc. IFR Int. Symposium on Robotics (ISR 2010).

Jain, A. and Kemp, C. (2010). El-e: an assistive mobile manipulator that autonomously fetches objects from flat surfaces. Autonomous Robots, 28:45-64.

Meeussen, W., Wise, M., Glaser, S., Chitta, S., McGann, C., Mihelich, P., Marder-Eppstein, E., Muja, M., Eruhimov, V., Foote, T., Hsu, J., Rusu, R. B., Marthi, B., Bradski, G., Konolige, K., Gerkey, B., and Berger, E. (2010). Autonomous door opening and plugging in with a personal robot. In Int. Conference on Robotics and Automation.

Micire, M., Desai, M., Drury, J. L., McCann, E., Norton, A., Tsui, K. M., and Yanco, H. A. (2011). Design and validation of two-handed multi-touch tabletop controllers for robot teleoperation. In Proc. 15th Int. Conf. on 